\title{
Evitando a pandemia da pobreza: possibilidades para 0 programa Bolsa Família e para o Cadastro Único em resposta à COVID-19
}

\section{Luis Henrique Paiva ${ }^{1}$}

Pedro H. G. Ferreira de Souza ${ }^{1}$

Letícia Bartholo ${ }^{1}$

Sergei Soares 1

1 Instituto de Pesquisa Econômica Aplicada / Diretoria de Estudos e Relações Econômicas e Políticas Internacionais, Brasília / DF - Brasil

Neste estudo propomos três medidas para potencializar o uso do Programa Bolsa Família e do Cadastro Único para Programas Sociais (Cadastro Único) como resposta às consequências econômicas da pandemia da COVID-19. As medidas propostas são as seguintes: (i) zerar a fila de espera do Programa e suspender procedimentos que possam levar à cessação de benefícios; (ii) reajustar benefícios e linha de elegibilidade do Programa; e (iii) conceder um benefício extraordinário para todas as famílias do Cadastro Único, beneficiárias ou não do Bolsa Família. As combinações para as alternativas destas medidas resultam em 56 cenários com parâmetros únicos, para os quais, com base nos dados do Cadastro Único e na folha de pagamentos do Bolsa Família, estimam-se o público alvo e os custos. Também avaliamos as eventuais dificuldades institucionais e operacionais para a implementação destas medidas, já que as iniciativas podem ter uma magnitude inédita na política social brasileira. Recomendamos uma combinação relativamente generosa destas medidas, por um período de pelo menos seis meses. Palavras-chave: COVID-19; pobreza; Bolsa Família; Cadastro Único.

\section{Evitando la pandemia de la pobreza: posibilidades para el "Programa Bolsa Família" y el Registro Único en respuesta a la COVID-19}

Este estudio propone tres medidas para potenciar el uso del "Programa Bolsa Família" y del Registro Único (Cadastro Único) para Programas Sociales como respuestas a las consecuencias económicas de la pandemia de COVID-19. Se propone (i) reducir a cero la lista de espera del programa y posponer los procedimientos administrativos que podrían resultar en recortes de beneficios; (ii) reajustar el umbral de elegibilidad y el valor de los beneficios del "Bolsa Família"; y (iii) otorgar un beneficio de emergencia, por al menos seis meses, a todas las familias inscritas en el Registro Único (sean o no beneficiarias del "Bolsa Família") cuyo ingreso per cápita sea inferior a la mitad del salario mínimo. Las combinaciones de las alternativas de estas medidas dan como resultado 56 escenarios diferentes. Sobre la base de los datos del Registro Único y la nómina del “Bolsa Família”, el estudio estima el público objetivo y los costos de las medidas propuestas. Dada la magnitud sin precedentes de estas medidas, el estudio también evalúa las posibles dificultades institucionales y operativas para su implementación. Se recomienda una combinación relativamente generosa de estas medidas por un período de al menos 6 meses.

Palabras clave: COVID-19; pobreza; Bolsa Família; Cadastro Único.

\section{Avoiding the poverty pandemic: the potential of the Bolsa Familia program and the Single Registry for Social Programs to face the COVID-19 pandemic}

This study suggests three measures to enhance the potential of the Bolsa Familia program and Single Registry for Social Protection (Cadastro Unico) as responses to the economic consequences of the COVID-19 pandemic. We propose to: (i) enroll all eligible households already in the Cadastro Unico in the Bolsa Familia program, and postpone all verification and recertification processes that could result in benefit cuts; (ii) adjust the eligibility thresholds of Bolsa Familia and the value of the benefits; and (iii) grant an emergency benefit, for at least six months, to all families enrolled in the Single Registry (regardless of whether they are Bolsa Familia beneficiaries or not) whose per capita income is below half a minimum wage. The combination of these measures results in 56 different scenarios. Based on the data from the Single Registry and the Bolsa Familia payroll, the study estimates the target audience and costs. Given the unprecedented magnitude of these measures, the study also emphasizes possible institutional and operational difficulties for their implementation. A combination of these measures is recommended, with a duration of at least six months. Keywords: COVID-19; poverty; Bolsa Família; Cadastro Único. 


\section{INTRODUÇÃO}

O objetivo deste trabalho é propor maneiras de potencializar o uso do Programa Bolsa Família (PBF) e do Cadastro Único para Programas Sociais (Cadastro Único) como mecanismos de redução dos impactos econômicos e sociais da pandemia da COVID-19. As propostas foram elaboradas por meio de combinações de medidas, que resultam em 56 diferentes simulações.

As transferências condicionadas de renda (classe de programas da qual o PBF faz parte) surgiram no Brasil, em nível local, em meados da década de 1990 e, nacionalmente, no início dos anos 2000, junto com o Cadastro Único, o principal registro administrativo dos programas brasileiros voltados para famílias de baixa renda (Lindert, Linder, Hobbs, \& De La Brière, 2007; Paiva, Cotta, \& Barrientos, 2019).

A focalização e o impacto na pobreza do PBF foram largamente estudados. As conclusões podem ser resumidas da seguinte maneira: o PBF é a transferência mais progressiva (isto é, a que mais contribui para a redução das desigualdades) feita pelo Governo Federal (Hoffman, 2013; Instituto de Pesquisa Econômica Aplicada [IPEA], 2012); sua introdução levou a sinais de ruptura no ciclo de pobreza (Pena, Pinheiro, Albuquerque, \& Fernandes, 2015) e seus impactos na redução da extrema pobreza são limitados apenas pelo baixo valor das transferências (Osório, Soares, \& Souza, 2011; Paiva, Souza, \& Nunes, 2020).

Estas características fazem do PBF e do Cadastro Único instrumentos ímpares na mitigação dos impactos econômicos e, consequentemente, sociais da pandemia da COVID-19. O Instituto Brasileiro de Economia da Fundação Getulio Vargas (FGV IBRE, 2020) estima a queda do PIB e da renda das famílias, neste ano, em respectivamente 3,4\% e 4,0\%. A queda do PIB, entretanto, está sendo rapidamente ajustada pelo Governo e pelo mercado: em seu Boletim Macrofiscal divulgado em 13 de maio, a Secretaria de Política Econômica (SPE) do Ministério da Economia (2020) estimou a queda do PIB em 4,70\%; por sua vez, o mercado, tal como captado pelo Focus - Relatório de Mercado, de 15 de maio (Banco Central do Brasil [Bacen], 2020), já registrava projeção para o PIB de -5,12\% (ante $-2,96 \%$, feita quatro semanas antes). Os indicadores de mercado de trabalho da Pesquisa Nacional por Amostra de Domicílios Contínua (Pnad Contínua/Instituto Brasileiro de Geografia e Estatística [IBGE]) divulgados para o primeiro trimestre ainda não captaram os impactos da crise econômica sobre o desemprego e a renda, mas a farta evidência pontual destacada pela mídia sugere que estamos diante de uma crise econômica vasta, profunda e com impactos sociais potencialmente devastadores.

Antes de prosseguirmos, acrescentamos três considerações. Primeiro, há precedente local e internacional para o uso de programas de transferências como resposta a situações emergenciais e/ou humanitárias. O aluguel social no Rio de Janeiro em 2011 e o benefício extraordinário pago aos moradores de Brumadinho em 2019 são exemplos locais. Levantamento feito pelo economista Ugo Gentilini (2020), do Banco Mundial, indica que cerca de 60 países já utilizam alguma forma de transferência de renda como parte das respostas à COVID-19. Em outras palavras, transferências de renda têm sido consideradas internacionalmente uma medida fundamental para mitigar o aumento da pobreza estrutural e conjuntural produzido pela pandemia.

Segundo, as sugestões aqui efetuadas partem do modelo operacional do PBF e do Cadastro Único. Isso implica que as sugestões abrangem mais de $30 \%$ da população brasileira cuja renda familiar mensal é inferior a $1 \frac{1}{2}$ salário mínimo por pessoa $(\mathrm{R} \$ 522,50)$.

Terceiro, em qualquer resposta à COVID-19 de suporte financeiro às famílias de baixa renda, devem ser consideradas dificuldades institucionais e operacionais. Este é o critério aqui adotado: 
todas as sugestões feitas são de operação viável em curto período e as propostas são apresentadas em ordem crescente de dificuldade institucional e operacional.

Doravante, o trabalho está organizado da seguinte forma: a seção 2 apresenta a simulação das três medidas; a seção 3 descreve os principais cenários detalhadamente e relata os resultados obtidos em termos de escopo e custos; e a seção 4 indica os cenários mais recomendados dentre os 56 simulados. Por fim, o apêndice traz os números obtidos para cada uma das simulações.

\section{CRITÉRIOS E CENÁRIOS DE INTERVENÇÃO}

Três medidas são aqui apresentadas; duas delas contêm mais de um cenário.

\section{A. Zerar a fila do PBF e postergar os processos de averiguação e revisão cadastral}

Medida essencial, por dois motivos: (i) permite a proteção monetária de todas as famílias que estão à espera de ingressar no PBF e (ii) evita que as famílias se dirijam desnecessariamente aos Centros de Referência em Assistência Social (CRAS) para atualização de suas informações do Cadastro Único. Como os CRAS estarão na linha de frente da resposta assistencial à crise, o ideal é reduzir ou eliminar demandas de cadastramento que não forem absolutamente necessárias.

\section{B. Reajustar a linha de elegibilidade e o valor dos benefícios do PBF}

Como não há nenhuma indexação no PBF, o valor real das linhas de elegibilidade e dos benefícios depende de reajustes discricionários que não têm sido suficientes para manter o poder de compra.

C. Criar um benefício extraordinário por pelo menos seis meses para todas as famílias do Cadastro Único (beneficiárias ou não do PBF) com renda familiar per capita inferior a meio salário mínimo

Diante da necessidade de distanciamento social, a concessão de benefícios temporários para famílias vulneráveis é uma resposta preventiva e mais racional do que simplesmente esperar que essas famílias fiquem abaixo da linha de pobreza e se dirijam aos CRAS para atualizar suas informações cadastrais. Famílias com cadastro atualizado e renda per capita inferior a meio salário mínimo mesmo que não sejam beneficiárias do PBF - estão sob forte risco de se tornarem pobres e, por isso, fazem jus a um benefício extraordinário temporário. As famílias que já estão no programa receberiam tanto o benefício ordinário regular quanto o benefício extraordinário.

As simulações foram feitas com base na extração dos dados do Cadastro Único de dezembro de 2018 (última extração do Cadastro disponível no IPEA) e da folha de pagamentos do PBF de janeiro de 2019.

As extrações do Cadastro Único, realizadas mensalmente pela Caixa Econômica Federal (CAIXA) e pelo Ministério da Cidadania, são o mais rico registro administrativo para estudos sobre políticas sociais. O Cadastro Único capta um amplo conjunto de informações pessoais (documentação, escolaridade, trabalho e renda) e domiciliares (composição familiar, características do domicílio, acesso a serviços de utilidade pública), fundamentais para a implementação e gestão de políticas sociais nos três níveis da federação.

Por suas características, o Cadastro Único é a mais precisa fonte de informação para estimativas de impacto orçamentário de alterações de políticas nele baseadas, como as feitas neste trabalho. Como se tem acesso ao universo dos beneficiários ou potenciais beneficiários e os registros sofrem alterações muito lentas ao longo do tempo, essas estimativas tendem a ser muito precisas. 
$\mathrm{Na}$ folha de pagamentos do Bolsa Família de janeiro de 2019, havia 13,76 milhões de famílias e 42,73 milhões de pessoas. Somadas aos não beneficiários com cadastro atualizado no Cadastro Único de dezembro de 2018, tem-se 21,14 milhões de famílias e 63,60 milhões de pessoas.

Os exercícios realizados na próxima seção consistiram fundamentalmente em estimar, com base nos microdados acima descritos, (i) as famílias não beneficiárias que se adéquam aos critérios de elegibilidade do Programa e (ii) o custo da concessão dos benefícios do Programa (Medidas A e B) e do benefício extraordinário (Medida C). Foram considerados os critérios de elegibilidade vigentes (para a Medida A) e os critérios de elegibilidade propostos (para os casos das Medidas B e C).

As estimativas obtidas foram comparadas ao cenário base, para que o leitor tenha a noção precisa do esforço fiscal necessário à implementação de cada uma das medidas e suas combinações. A seção 3 apresenta algumas destas combinações, e o apêndice, a totalidade das estimativas para cada um dos 56 cenários.

\section{CENÁRIOS BASEADOS NA ESTRUTURA DO PBF}

\subsection{Medida A: zerar a fila do PBF e postergar os processos de averiguação e revisão cadastral}

O PBF possui uma fila estimada de aproximadamente 1,7 milhão de famílias. A concessão de benefícios para todas as famílias elegíveis do Cadastro Único elevaria o público do Programa para 15,5 milhões de famílias.

Se esta medida tivesse sido adotada para o mês de abril e estendida até o final do ano, seu impacto estaria na casa de $\mathrm{R} \$ 2,24$ bilhões, um aumento de quase $10 \%$ em relação ao cenário atual (cf. Tabela 1). Com R 3 bilhões, estima-se também ser possível interromper os processos de averiguação e revisão cadastral (e, com isso, bloqueios, suspensões e cancelamentos) ao longo dos próximos meses.

\section{TABELA 1 ESCOPO E CUSTO DA MEDIDA A}

\begin{tabular}{lcc|c|c|c|c|c|} 
Cenários & \multicolumn{2}{c}{ Escopo (milhões) } & Benefício médio & \multicolumn{2}{c}{ Custo (R\$ bilhões) } & \multicolumn{2}{c}{ Variação total } \\
& Famílias & Pessoas & por família (R\$) & Por mês & Total & Absoluta (R\$ bi) & Relativa (\%) \\
\hline Cenário atual & 13,8 & 42,7 & 188 & 2,586 & 23,273 & - & - \\
A. Fila zero & 15,5 & 47,7 & 183 & 2,835 & 25,513 & 2,240 & 9,6 \\
\hline
\end{tabular}

N.B.: O custo total diz respeito aos nove meses restantes de 2020 (abril a dezembro).

Fonte: Elaborada pelos autores com base no Cadastro Único (dezembro/2018) e na Folha de Pagamentos do PBF (janeiro/2019).

Nível das dificuldades institucionais: virtualmente inexistentes. A concessão é decisão administrativa, assim como a postergação dos processos de averiguação e revisão.

Nível das dificuldades operacionais: a emissão e a distribuição de cartões são procedimentos operacionais bem estabelecidos, mas a escala (de 1,7 milhão) é incomum, exigindo atenção. Os saques 
podem ser feitos diretamente nas agências da Caixa, mediante identificação, mas isso é desaconselhável do ponto de vista operacional e sanitário.

\subsection{Medida B: reajustar a linha de elegibilidade e o valor dos benefícios}

Para a medida B, apresentamos três cenários de reajustes.

B1) Por um percentual que leve as linhas de elegibilidade a voltarem ao valor da sua média histórica. Com isso, a linha de extrema pobreza iria a $\mathrm{R} \$ 105$, mesmo valor do benefício básico e referência para o cálculo do benefício de superação da pobreza extrema (BSP); a linha de pobreza subiria para $\mathrm{R} \$ 210$; benefícios variáveis para grávidas, nutrizes e crianças iriam a $\mathrm{R} \$$ 48 e o benefício variável vinculado ao adolescente (BVJ), a R \$ 57. O percentual do reajuste é de aproximadamente $18 \%$.

B2) Por um percentual que leve as linhas de elegibilidade a voltarem ao valor real que tinham em janeiro de 2004. A linha de extrema pobreza iria a R\$115, mesmo valor do benefício básico e referência para o BSP; a linha de pobreza, a R 230 ; benefícios variáveis iriam a R 53 e o BVJ, a $\mathrm{R} \$ 62$. O percentual de reajuste é de aproximadamente $29 \%$.

B3) Por um percentual que faça as linhas voltarem ao valor real do seu pico histórico, observado em agosto de 2009. A linha de extrema pobreza iria a $\mathrm{R} \$ 125$, mesmo valor do benefício básico e referência para o BSP; a linha de pobreza a $\mathrm{R} \$ 250$; benefícios variáveis iriam a R\$ 58 e o BVJ, a $\mathrm{R} \$ 67$. O percentual de reajuste é próximo a 41\%. Mesmo com esse reajuste, a linha de extrema pobreza do PBF permaneceria abaixo da linha de PPC \$1,90 por dia (isso é, de USD 1,90 por dia, convertido pela Paridade do Poder de Compra, ou aproximadamente R $\$ 150$ por mês), que toma por referência as linhas de alguns dos países mais pobres do mundo.

O impacto orçamentário ocorreria por duas vias: aumentaria o número de famílias elegíveis e elevaria o valor dos benefícios. Apresentamos na Tabela 2 o impacto completo da medida, isto é, a inclusão de todas as famílias elegíveis, de acordo com as novas linhas de elegibilidade, bem como o custo efetivo com o aumento dos benefícios, o que pode ser entendido como o impacto combinado das Medidas A e B. O impacto independente dos cenários da Medida B está no apêndice deste trabalho.

Os cenários AB1-AB3 aumentariam a clientela do PBF entre 17\% e 21\%, enquanto os benefícios médios por família cresceriam entre 15\% e 38\%. No cenário AB3, seriam incorporadas quase 3 milhões de famílias ao PBF, ou seja, a proteção do programa seria estendida para quase 9 milhões de pessoas que hoje estão desprotegidas.

Com isso, o impacto orçamentário ficaria entre $\mathrm{R} \$$ 8,0 bilhões (cenário $A B 1$ ) e $\mathrm{R} \$ 15,6$ bilhões (AB3). O da alternativa intermediária seria $\mathrm{R} \$ 11,6$ bilhões. O custo do Programa no resto do ano (de abril a dezembro) seria entre 1/3 e 2/3 maior do que no cenário atual. 
TABELA 2 ESCOPO E CUSTO DOS CENÁRIOS DA MEDIDA B COMBINADOS COM A MEDIDA A

\begin{tabular}{|c|c|c|c|c|c|c|c|}
\hline \multirow{2}{*}{ Cenários } & \multicolumn{2}{|c|}{ Escopo (milhões) } & \multirow{2}{*}{$\begin{array}{l}\text { Benefício médio } \\
\text { por família (R\$) }\end{array}$} & \multicolumn{2}{|c|}{ Custo (R\$ bilhões) } & \multicolumn{2}{|c|}{ Variação total } \\
\hline & Famílias & Pessoas & & Por mês & Total & Absoluta (R\$ bi) & Relativa (\%) \\
\hline Cenário atual & 13,8 & 42,7 & 188 & 2,586 & 23,273 & - & - \\
\hline $\begin{array}{l}A+B 1 . \text { Reajuste } \\
\text { de aprox. } 18 \%\end{array}$ & 16,0 & 49,5 & 217 & 3,474 & 31,265 & 7,992 & 34,3 \\
\hline $\begin{array}{l}A+B 2 . \text { Reajuste } \\
\text { de aprox. } 29 \%\end{array}$ & 16,2 & 50,1 & 239 & 3,873 & 34,856 & 11,583 & 49,8 \\
\hline $\begin{array}{l}\text { A + B3. Reajuste } \\
\text { de aprox. } 41 \%\end{array}$ & 16,6 & 51,6 & 260 & 4,314 & 38,826 & 15,553 & 66,8 \\
\hline
\end{tabular}

N.B.: O custo total diz respeito aos nove meses restantes de 2020 (abril a dezembro).

Fonte: Elaborada pelos autores com base no Cadastro Único (dezembro/2018) e na Folha de Pagamentos do PBF (janeiro/2019).

Nível das dificuldades institucionais: baixo. A linha de elegibilidade e o valor dos benefícios são reajustados via decreto, sem necessidade de alteração legal.

Nível das dificuldades operacionais: para as famílias já beneficiárias, não há dificuldade operacional. A inclusão de novas famílias implica emissão e envio de cartões, como abordado anteriormente. Há potencial de atrito, mas o processo operacional já está bem definido.

\subsection{Medida C: Criar um benefício extraordinário temporário para todas as famílias do Cadastro Único com renda familiar per capita inferior a meio salário mínimo}

A medida $C$ prevê a criação de benefícios extraordinários que seriam pagos tanto para famílias beneficiárias do PBF (que continuariam recebendo seus benefícios ordinários), quanto para as demais famílias do Cadastro Único com informações atualizadas, com renda familiar per capita inferior a meio salário mínimo, isto é, R\$ 522,50.

Simulamos seis tipos diferentes de benefícios extraordinários fixos com duração de seis meses:

C1) R $\$ 150$ por família;

C2) R\$ 300 por família;

C3) R\$ 450 por família;

C4) R $\$ 50$ per capita;

C5) R\$ 100 per capita;

C6) R\$ 150 per capita.

Com o benefício extraordinário, um maior número de pessoas passaria a contar com alguma renda em um momento de forte impacto negativo na economia. Caso a crise social provocada pela COVID-19 dure mais do que esse período, os benefícios extraordinários podem e devem ser prorrogados. 
A Tabela 3 apresenta os resultados da simulação dos cenários $\mathrm{C} 1$ a C6 em conjunto com a medida $\mathrm{A}$. Os resultados isolados dos cenários C1 a C6 estão no apêndice.

Os cenários simulados na Tabela 3 apresentam alcance maior do que qualquer simulação anterior, visto que os benefícios extraordinários são pagos a todas as 21,1 milhões de famílias com renda abaixo de meio salário mínimo per capita registradas no Cadastro Único. ${ }^{1}$ Nesses cenários, o público atingido pelas transferências assistenciais aumentaria em cerca de 50\%, estendendo um nível mínimo de proteção social a aproximadamente 63,6 milhões de pessoas, ou seja, pouco mais de $30 \%$ da população brasileira.

Como as famílias do Cadastro Único têm em média três componentes, os números escolhidos tornam os impactos orçamentários dos cenários C1-C3 e C4-C6 muito semelhantes. Em tese, os cenários C4-C6 seriam mais justos, pois transfeririam benefícios com o mesmo valor per capita para todas as famílias. Contudo há risco de dificuldades operacionais consideráveis para a implementação de um benefício desse tipo. Retornaremos a esse ponto mais adiante.

Seja como for, o benefício médio por família aumentaria fortemente. Nos cenários mais conservadores $(\mathrm{A}+\mathrm{C} 1$ e A + C4), a elevação seria de pouco mais de 50\%. Nos cenários mais ousados (A + C3 e A + C6), o acréscimo chegaria a mais de $210 \%$, com benefício médio de cerca de $\mathrm{R} \$ 585$ por família.

Como há tanto expansão de cobertura quanto aumento do valor médio, o aumento relativo do custo nos nove meses restantes do ano oscila entre $91 \%$ e $256 \%$, mesmo considerando a duração temporária do benefício extraordinário. Em termos absolutos, o custo adicional ficaria entre $\mathrm{R} \$ 21$ bilhões e $\mathrm{R} \$ 59$ bilhões. Apesar da magnitude, vale ressaltar que, mesmo no cenário mais generoso, as transferências assistenciais sairiam de $0,4 \%$ para 1,2\% do PIB, valor compatível com o registrado em tempos normais em muitos países desenvolvidos.

\section{TABELA 3 ESCOPO E CUSTO DOS CENÁRIOS DA MEDIDA C COMBINADOS COM A MEDIDA A}

\begin{tabular}{|c|c|c|c|c|c|c|c|}
\hline \multirow{2}{*}{ Cenários } & \multicolumn{2}{|c|}{ Escopo (milhões) } & \multirow{2}{*}{$\begin{array}{c}\text { Benefício } \\
\text { médio por } \\
\text { família (R\$) }\end{array}$} & \multicolumn{2}{|c|}{ Custo (R\$ bilhões) } & \multicolumn{2}{|c|}{ Variação total } \\
\hline & Famílias & Pessoas & & Por mês & Total & $\begin{array}{l}\text { Absoluta } \\
\text { R\$ bi) }\end{array}$ & Relativa (\%) \\
\hline Cenário atual & 13,8 & 42,7 & 188 & 2,586 & 23,273 & - & - \\
\hline $\begin{array}{l}\mathrm{A}+\mathrm{C} 1(\mathrm{R} \$ 150 \text { por } \\
\text { família) }\end{array}$ & 21,1 & 63,6 & 284 & 6,005 & 44,536 & 21,263 & 91,4 \\
\hline $\begin{array}{l}A+C 2(R \$ 300 \text { por } \\
\text { família) }\end{array}$ & 21,1 & 63,6 & 434 & 9,176 & 63,558 & 40,286 & 173,1 \\
\hline
\end{tabular}

${ }^{1}$ Números referentes à situação do CadÚnico em dezembro de 2018. 


\begin{tabular}{|c|c|c|c|c|c|c|c|}
\hline \multirow{2}{*}{ Cenários } & \multicolumn{2}{|c|}{ Escopo (milhões) } & \multirow{2}{*}{$\begin{array}{l}\text { Benefício } \\
\text { médio por } \\
\text { família (R\$) }\end{array}$} & \multicolumn{2}{|c|}{ Custo (R\$ bilhões) } & \multicolumn{2}{|c|}{ Variação total } \\
\hline & Famílias & Pessoas & & Por mês & Total & $\begin{array}{l}\text { Absoluta } \\
\text { R\$ bi) }\end{array}$ & Relativa (\%) \\
\hline $\begin{array}{l}\text { A + C3 (R\$ } 450 \text { por } \\
\text { família) }\end{array}$ & 21,1 & 63,6 & 584 & 12,346 & 82,581 & 59,309 & 254,8 \\
\hline$A+C 4(\mathrm{R} \$ 50$ per capita) & 21,1 & 63,6 & 285 & 6,015 & 44,591 & 21,319 & 91,6 \\
\hline $\begin{array}{l}\text { A + C5 (R\$ } 100 \text { per } \\
\text { capita) }\end{array}$ & 21,1 & 63,6 & 435 & 9,194 & 63,670 & 40,398 & 173,6 \\
\hline $\begin{array}{l}\text { A + C6 (R\$ } 150 \text { per } \\
\text { capita) }\end{array}$ & 21,1 & 63,6 & 585 & 12,374 & 82,749 & 59,476 & 255,6 \\
\hline
\end{tabular}

N.B.: O custo total diz respeito aos nove meses restantes de 2020 (abril a dezembro). Como os benefícios extraordinários são previstos por apenas seis meses, o custo total de cada cenário considera o custo mensal de seis meses de cada um e o valor previsto na medida A para os três meses finais do ano. O benefício médio por família foi calculado usando o custo mensal dos cenários.

Fonte: Elaborada pelos autores com base no Cadastro Único (dezembro/2018) e na Folha de Pagamentos do PBF (janeiro/2019).

A combinação entre as medidas A, B e C gera 18 possíveis cenários (três cenários da medida $\mathrm{B}$ multiplicado por seis cenários da medida C). Para simplificar, optamos por considerar apenas o resultado da combinação entre as medidas A, B2 e C1-C3, visto que a medida B2 é o ponto intermediário entre B1 e B3 e os impactos orçamentários de C4, C5 e C6 são praticamente idênticos aos de C1, C2 e C3. Os números para todas as combinações são reportados no apêndice.

A Tabela 4 exibe as estimativas para as combinações escolhidas. O público é idêntico ao da Tabela 3 , as diferenças ficam por conta dos valores médios dos benefícios e seus impactos orçamentários.

Os benefícios médios subiriam entre $77 \%$ e $237 \%$. Neste último caso (cenário A + B2 + C3), cada família receberia em média $\mathrm{R} \$ 633$ enquanto durar o benefício extraordinário. Em termos per capita, isso significa um benefício médio de $\mathrm{R} \$ 210$ por pessoa durante pelo menos seis meses, valor mais alto dentre todos os apresentados neste texto. Depois dos seis meses iniciais, cerca de 4,9 milhões de famílias deixariam de receber qualquer benefício, enquanto, dentre as famílias remanescentes, cada uma receberia os benefícios reajustados do PBF com valor médio de R \$239 (ou R\$ 77 por pessoa).

O impacto orçamentário variaria entre $\mathrm{R} \$ 30,6$ bilhões $(\mathrm{A}+\mathrm{B} 2+\mathrm{C} 1)$ e $\mathrm{R} \$ 68,7$ bilhões $(\mathrm{A}+$ B2 + C3) até o fim do ano. Em comparação com o cenário atual, todas as simulações mais do que dobram o orçamento, chegando, no limite, quase a triplicá-lo. O custo total com transferências (incluindo os meses de janeiro a março) subiria de $0,4 \%$ para algo entre $0,8 \%$ e $1,4 \%$ do PIB. 


\section{TABELA 4}

\section{ESCOPO E CUSTO DOS CENÁRIOS C1-C3 COMBINADOS COM A MEDIDA A E COM O CENÁRIO B2}

\begin{tabular}{|c|c|c|c|c|c|c|c|}
\hline \multirow[b]{2}{*}{ Cenários } & \multicolumn{2}{|c|}{ Escopo (milhões) } & \multirow{2}{*}{$\begin{array}{l}\text { Benefício } \\
\text { médio por } \\
\text { família }(\mathrm{R} \$)\end{array}$} & \multicolumn{2}{|c|}{ Custo (R\$ bilhões) } & \multicolumn{2}{|c|}{ Variação total } \\
\hline & Famílias & Pessoas & & Por mês & Total & $\begin{array}{c}\text { Absoluta } \\
\text { (R\$ bi) }\end{array}$ & $\begin{array}{c}\text { Relativa } \\
(\%)\end{array}$ \\
\hline Cenário atual & 13,8 & 42,7 & 188 & 2,586 & 23,273 & - & - \\
\hline $\begin{array}{l}\text { A + B2 (reaj. 29\%) + C1 (R\$ } \\
\text { 150/ffamília) }\end{array}$ & 21,1 & 63,6 & 333 & 7,043 & 53,879 & 30,606 & 131,5 \\
\hline $\begin{array}{l}\text { A + B2 (reaj. 29\%) + C2 (R\$ } \\
\text { 300/família) }\end{array}$ & 21,1 & 63,6 & 483 & 10,214 & 72,902 & 49,629 & 213,2 \\
\hline $\begin{array}{l}\text { A + B2 (reaj. 29\%) + C3 (R\$ } \\
\text { 450/família) }\end{array}$ & 21,1 & 63,6 & 633 & 13,384 & 91,925 & 68,652 & 295,0 \\
\hline
\end{tabular}

N.B.: O custo total diz respeito aos nove meses restantes de 2020 (abril a dezembro). Como os benefícios extraordinários são previstos por apenas seis meses, o custo total de cada cenário considera o custo mensal de seis meses de cada um e o valor previsto no cenário A + B2 para os três meses finais do ano. O benefício médio por família foi calculado usando o custo mensal dos cenários.

Fonte: Elaborada pelos autores com base no Cadastro Único (dezembro/2018) e na Folha de Pagamentos do PBF (janeiro/2019).

Nível das dificuldades institucionais: intermediário. Exige edição e aprovação de Medida Provisória (MP). Recomenda-se legislação específica, sem associação com a legislação do PBF, pois o benefício extraordinário teria público mais amplo. O ideal é que esse benefício fizesse, tanto quanto possível, uso da estrutura operacional do PBF.

Nível das dificuldades operacionais: alto. O Sistema de Benefícios ao Cidadão (SIBEC), da Caixa, não está preparado para gerar um benefício desta natureza. Criar um benefício fora das linhas de elegibilidade do PBF requer especificar um novo processo de seleção de beneficiários e gerar uma nova folha de pagamentos.

Por razões operacionais, sugere-se que seja adotado o benefício com valor único por família, pois há precedente (em Brumadinho). Isso reduziria as chances de erro na operacionalização do benefício.

Ainda que se consiga gerar uma folha no início de abril, para pagamento na segunda quinzena do mês, possíveis problemas com os meios de pagamento (envolvendo emissão e envio de milhões de cartões ou a criação automática de contas digitais) podem levar a atrasos no acesso efetivo ao benefício.

A possibilidade de efetuar o saque presencial na rede da Caixa deve ser avaliada em virtude das ações de contenção da COVID-19.

Outra opção seria o depósito direto em conta corrente ou poupança (para beneficiários clientes da Caixa); ou o uso de eventual cartão-cidadão já existente (ex-beneficiários do PBF, do abono salarial ou do seguro-desemprego). Seja como for, qualquer medida que vá além dos limites do PBF enfrentará dificuldades operacionais. 


\section{RECOMENDAÇÃO}

A pandemia da COVID-19 traz desafios inéditos ao sistema brasileiro de proteção social. Trabalhadores informais, desempregados e famílias pobres estão expostos à combinação de pandemia e recessão. Dada a probabilidade de desdobramentos catastróficos do ponto de vista social, nossa recomendação tende para os cenários mais generosos. Na pior das hipóteses, mesmo se os riscos sociais estiverem superestimados, a despesa adicional seria quase toda temporária e não chegaria a 1,5\% do PIB.

Neste contexto, recomendamos que, no mínimo, o cenário A + B2 + C3 seja implementado. Em outras palavras, sugerimos que:

- A fila de espera para receber o PBF seja eliminada e os processos de averiguação e revisão cadastral sejam suspensos;

- As linhas de elegibilidade e os benefícios do PBF sejam reajustados em aproximadamente 29\%;

- Seja criado um benefício extraordinário, por seis meses, prorrogáveis, no valor de R\$ 450 por família, para todas as famílias do Cadastro Único com renda familiar per capita inferior a meio salário mínimo (R 522,50$)$.

Nesse caso, os $30 \%$ mais pobres da população brasileira poderiam contar com uma renda mensal de pelo menos R $\$ 450$ por família. Beneficiários do PBF teriam em média uma segurança de renda mensal de quase R $\$ 690$ por família. Após o fim do benefício extraordinário, os beneficiários continuariam recebendo em média $\mathrm{R} \$ 240$ por família (isto é, $\mathrm{R} \$ 77$ per capita), valor $27 \%$ maior do que o pago atualmente.

O impacto orçamentário em 2020 seria de R $\$ 68,6$ bilhões. Porém mais de 80\% desse impacto seria temporário. Para 2021, o aumento de despesa seria modesto - apenas R \$11,6 bilhões, menos de $0,2 \%$ do PIB brasileiro de 2019.

Recomendamos também que o benefício extraordinário seja estendido pelo tempo necessário para a superação da crise social.

Considerando-se a defasagem do poder de compra dos benefícios atuais do PBF e os riscos econômicos e sociais decorrentes da pandemia da COVID-19, parece-nos um preço baixo a pagar para garantir um nível mínimo de bem-estar aos mais pobres. 


\section{REFERÊNCIAS}

Banco Central do Brasil. (2020, 15 de maio). Focus - Relatório de Mercado. Brasília, DF: Bacen, 2020. Recuperado de https://www.bcb.gov.br/publicacoes/ focus

Gentilini, U. (2020, 15 de março). 14 countries have so far used some form of \#castransfer and \#basicincome program as \#coronavirus response. Twitter.com. Recuperado de https://twitter.com/ Ugentilini/status/1239184435402211336

Hoffman, R. (2013). Transferências de renda e desigualdade no Brasil (1995-2011). In T. Campello, \& M. NERI (Orgs.), Programa Bolsa Família - uma década de inclusão e cidadania. Brasília, DF: Instituto de Pesquisa Econômica Aplicada.

Instituto Brasileiro de Economia da Fundação Getulio Vargas. (2020, abril). Boletim Macro. Rio de Janeiro, RJ: FGV. Recuperado de https:// portalibre.fgv.br/sites/default/files/2020-05/ boletimmacroibre_2004.pdf

Instituto de Pesquisa Econômica Aplicada. (2012). A década inclusiva (2001-2011): desigualdade, pobreza e políticas de renda. (Comunicados do Ipea, n. 155). Brasília, DF: Autor.

Lindert, K., Linder, A., Hobbs, J., \& De La Brière, B. (2007). The nuts and bolts of Brazil's Bolsa Família Program: implementing conditional cash transfers in a decentralized context. (Social Protection Discussion Paper, n. 709). Washington, DC: The World Bank.

Ministério da Economia. (2020, maio). Boletim Macrofiscal da SPE. Brasília, DF: Autor. Recuperado de https://static.poder360.com.br/2020/05/ Apresentacao-Boletim-MacroFiscal-Maio.pdf

Osório, R. G., Soares, S., \& Souza, P. F. (2011). Erradicar a pobreza extrema: um objetivo ao alcance do Brasil (Texto para Discussão, n. 1619). Brasília, DF: IPEA.

Paiva, L. H., Cotta, T., \& Barrientos, A. Brazil's Bolsa Familia Programme. (2019). In M. Compton, \& P. T. Hart (Org.), Great Policy Successes (pp. 1-24). Oxford, UK: Oxford University Press. Recuperado de https:// doi.org/10.1093/oso/9780198843719.001.0001

Paiva, L. H., Souza, M. F., \& Nunes, H. M. P. (2020, March). Targeting in the Bolsa Familia programme from 2012 to 2018 based on data from the Continuous National Household Sample Survey. (One Pager, n. 436). Brasília, DF: Institute for Applied Economic Research. Recuperado de https://ipcig.org/pub/ eng/OP436_Targeting_in_the_Bolsa_FamIlia_ programme_from_2012_to_2018.pdf

Pena, C. R., Pinheiro, D. S., Albuquerque, P. H., \& Fernandes, L. M. (2015). A eficácia das transferências de renda: as tendências da desigualdade antes e depois do Programa Bolsa Família. Revista de Administração Pública, 49(4), 889-913. 


\section{Luis Henrique Paiva}

10

https://orcid.org/0000-0001-9197-7332

Doutor em Sociologia; Coordenador de Estudos e Pesquisa em Seguridade Social da Diretoria de Estudos e Políticas Sociais do Instituto de Pesquisa Econômica Aplicada. E-mail: luis.paiva@ipea.gov.br

\section{Pedro H. G. Ferreira de Souza}

https://orcid.org/0000-0002-5271-0165

Doutor em Sociologia; Coordenador de Estudos e Pesquisas de Gestão de Informações e de Estudos sobre Pobreza e Desigualdade Social da Diretoria de Estudos e Políticas Sociais do Instituto de Pesquisa Econômica Aplicada. E-mail: pedro.ferreira@ipea.gov.br

\section{Letícia Bartholo}

iD

https://orcid.org/0000-0001-8340-0074

Mestre em Demografia; Especialista em Políticas Públicas e Gestão Governamental, em exercício na Diretoria de Estudos e Políticas Sociais do Instituto de Pesquisa Econômica Aplicada. E-mail: leticia.bartholo@ipea.gov.br

\section{Sergei Soares}

https://orcid.org/0000-0003-4806-0267

Doutor em Economia; Coordenador de Estudos em Instituições e Governança Internacional da Diretoria de Estudos e Relações Econômicas e Políticas Internacionais do Instituto de Pesquisa Econômica Aplicada. E-mail: sergei.soares@ipea.gov.br 
RAP | Evitando a pandemia da pobreza: possibilidades para o programa Bolsa Família e para o Cadastro Único em resposta à COVID-19

\section{APÊNDICE}

TABELA A RESULTADOS DOS 56 CENÁRIOS SIMULADOS

\begin{tabular}{|c|c|c|c|c|c|c|}
\hline \multirow[t]{2}{*}{ Número } & \multirow[t]{2}{*}{ Cenário } & \multicolumn{2}{|c|}{ Escopo (milhões) } & \multirow{2}{*}{$\begin{array}{c}\text { Custo por mês } \\
\text { (R\$ bilhões) }\end{array}$} & \multicolumn{2}{|c|}{$\begin{array}{l}\text { Benefício médio } \\
\text { mensal (R\$) }\end{array}$} \\
\hline & & Famílias & Pessoas & & Por família & Por pessoa \\
\hline 1 & Benchmark & 13,761 & 42,732 & 2,586 & 188 & 61 \\
\hline 2 & $A$ & 15,482 & 47,658 & 2,835 & 183 & 59 \\
\hline 3 & B1 & 13,761 & 42,732 & 3,108 & 226 & 73 \\
\hline 4 & B2 & 13,761 & 42,732 & 3,446 & 250 & 81 \\
\hline 5 & B3 & 13,761 & 42,732 & 3,798 & 276 & 89 \\
\hline 6 & $\mathrm{C} 1$ & 21,137 & 63,596 & 5,756 & 272 & 91 \\
\hline 7 & C2 & 21,137 & 63,596 & 8,927 & 422 & 140 \\
\hline 8 & C3 & 21,137 & 63,596 & 12,097 & 572 & 190 \\
\hline 9 & C4 & 21,137 & 63,596 & 5,766 & 273 & 91 \\
\hline 10 & C5 & 21,137 & 63,596 & 8,945 & 423 & 141 \\
\hline 11 & C6 & 21,137 & 63,596 & 12,125 & 574 & 191 \\
\hline 12 & $A+B 1$ & 16,045 & 49,488 & 3,474 & 217 & 70 \\
\hline 13 & $A+B 2$ & 16,221 & 50,150 & 3,873 & 239 & 77 \\
\hline 14 & $A+B 3$ & 16,605 & 51,599 & 4,314 & 260 & 84 \\
\hline 15 & $\mathrm{~A}+\mathrm{C} 1$ & 21,137 & 63,596 & 6,005 & 284 & 94 \\
\hline 16 & $\mathrm{~A}+\mathrm{C} 2$ & 21,137 & 63,596 & 9,176 & 434 & 144 \\
\hline 17 & $\mathrm{~A}+\mathrm{C} 3$ & 21,137 & 63,596 & 12,346 & 584 & 194 \\
\hline 18 & $\mathrm{~A}+\mathrm{C} 4$ & 21,137 & 63,596 & 6,015 & 285 & 95 \\
\hline 19 & $\mathrm{~A}+\mathrm{C} 5$ & 21,137 & 63,596 & 9,194 & 435 & 145 \\
\hline 20 & $\mathrm{~A}+\mathrm{C} 6$ & 21,137 & 63,596 & 12,374 & 585 & 195 \\
\hline 21 & $\mathrm{~B} 1+\mathrm{C} 1$ & 21,137 & 63,596 & 6,278 & 297 & 99 \\
\hline 22 & $\mathrm{~B} 1+\mathrm{C} 2$ & 21,137 & 63,596 & 9,449 & 447 & 149 \\
\hline 23 & $\mathrm{~B} 1+\mathrm{C} 3$ & 21,137 & 63,596 & 12,619 & 597 & 198 \\
\hline 24 & $\mathrm{~B} 1+\mathrm{C} 4$ & 21,137 & 63,596 & 6,288 & 297 & 99 \\
\hline 25 & $\mathrm{~B} 1+\mathrm{C} 5$ & 21,137 & 63,596 & 9,467 & 448 & 149 \\
\hline 26 & $\mathrm{~B} 1+\mathrm{C} 6$ & 21,137 & 63,596 & 12,647 & 598 & 199 \\
\hline 27 & $\mathrm{~B} 2+\mathrm{C} 1$ & 21,137 & 63,596 & 6,616 & 313 & 104 \\
\hline 28 & $\mathrm{~B} 2+\mathrm{C} 2$ & 21,137 & 63,596 & 9,787 & 463 & 154 \\
\hline
\end{tabular}




\begin{tabular}{|c|c|c|c|c|c|c|}
\hline \multirow[t]{2}{*}{ Número } & \multirow[t]{2}{*}{ Cenário } & \multicolumn{2}{|c|}{ Escopo (milhões) } & \multirow{2}{*}{$\begin{array}{l}\text { Custo por mês } \\
\text { (R\$ bilhões) }\end{array}$} & \multicolumn{2}{|c|}{$\begin{array}{l}\text { Benefício médio } \\
\text { mensal (R\$) }\end{array}$} \\
\hline & & Famílias & Pessoas & & Por família & Por pessoa \\
\hline 29 & $\mathrm{~B} 2+\mathrm{C} 3$ & 21,137 & 63,596 & 12,957 & 613 & 204 \\
\hline 30 & $\mathrm{~B} 2+\mathrm{C} 4$ & 21,137 & 63,596 & 6,626 & 313 & 104 \\
\hline 31 & $\mathrm{~B} 2+\mathrm{C} 5$ & 21,137 & 63,596 & 9,805 & 464 & 154 \\
\hline 32 & $\mathrm{~B} 2+\mathrm{C} 6$ & 21,137 & 63,596 & 12,985 & 614 & 204 \\
\hline 33 & $\mathrm{~B} 3+\mathrm{C} 1$ & 21,137 & 63,596 & 6,969 & 330 & 110 \\
\hline 34 & $\mathrm{~B} 3+\mathrm{C} 2$ & 21,137 & 63,596 & 10,139 & 480 & 159 \\
\hline 35 & $\mathrm{~B} 3+\mathrm{C} 3$ & 21,137 & 63,596 & 13,310 & 630 & 209 \\
\hline 36 & $\mathrm{~B} 3+\mathrm{C} 4$ & 21,137 & 63,596 & 6,978 & 330 & 110 \\
\hline 37 & B3+C5 & 21,137 & 63,596 & 10,158 & 481 & 160 \\
\hline 38 & $\mathrm{~B} 3+\mathrm{C} 6$ & 21,137 & 63,596 & 13,338 & 631 & 210 \\
\hline 39 & $\mathrm{~A}+\mathrm{B} 1+\mathrm{C} 1$ & 21,137 & 63,596 & 6,644 & 314 & 104 \\
\hline 40 & $\mathrm{~A}+\mathrm{B} 1+\mathrm{C} 2$ & 21,137 & 63,596 & 9,815 & 464 & 154 \\
\hline 41 & $\mathrm{~A}+\mathrm{B} 1+\mathrm{C} 3$ & 21,137 & 63,596 & 12,985 & 614 & 204 \\
\hline 42 & $\mathrm{~A}+\mathrm{B} 1+\mathrm{C} 4$ & 21,137 & 63,596 & 6,654 & 315 & 105 \\
\hline 43 & $\mathrm{~A}+\mathrm{B} 1+\mathrm{C} 5$ & 21,137 & 63,596 & 9,833 & 465 & 155 \\
\hline 44 & $\mathrm{~A}+\mathrm{B} 1+\mathrm{C} 6$ & 21,137 & 63,596 & 13,013 & 616 & 205 \\
\hline 45 & $\mathrm{~A}+\mathrm{B} 2+\mathrm{C} 1$ & 21,137 & 63,596 & 7,043 & 333 & 111 \\
\hline 46 & $\mathrm{~A}+\mathrm{B} 2+\mathrm{C} 2$ & 21,137 & 63,596 & 10,214 & 483 & 161 \\
\hline 47 & $\mathrm{~A}+\mathrm{B} 2+\mathrm{C} 3$ & 21,137 & 63,596 & 13,384 & 633 & 210 \\
\hline 48 & $\mathrm{~A}+\mathrm{B} 2+\mathrm{C} 4$ & 21,137 & 63,596 & 7,053 & 334 & 111 \\
\hline 49 & $\mathrm{~A}+\mathrm{B} 2+\mathrm{C} 5$ & 21,137 & 63,596 & 10,233 & 484 & 161 \\
\hline 50 & $\mathrm{~A}+\mathrm{B} 2+\mathrm{C} 6$ & 21,137 & 63,596 & 13,412 & 635 & 211 \\
\hline 51 & $\mathrm{~A}+\mathrm{B} 3+\mathrm{C} 1$ & 21,137 & 63,596 & 7,484 & 354 & 118 \\
\hline 52 & $\mathrm{~A}+\mathrm{B} 3+\mathrm{C} 2$ & 21,137 & 63,596 & 10,655 & 504 & 168 \\
\hline 53 & $\mathrm{~A}+\mathrm{B} 3+\mathrm{C} 3$ & 21,137 & 63,596 & 13,825 & 654 & 217 \\
\hline 54 & $\mathrm{~A}+\mathrm{B} 3+\mathrm{C} 4$ & 21,137 & 63,596 & 7,494 & 355 & 118 \\
\hline 55 & $\mathrm{~A}+\mathrm{B} 3+\mathrm{C} 5$ & 21,137 & 63,596 & 10,674 & 505 & 168 \\
\hline 56 & $A+B 3+C 6$ & 21,137 & 63,596 & 13,853 & 655 & 218 \\
\hline
\end{tabular}

Fonte: Elaborada pelos autores. 